\title{
Thermal state of transiently accreting neutron stars
}

\author{
D. G. Yakovlev ${ }^{1}$, K. P. Levenfish ${ }^{1}$, and P. Haensel ${ }^{2}$ \\ ${ }^{1}$ Ioffe Physical Technical Institute, Politekhnicheskaya 26, 194021 St.-Petersburg, Russia \\ 2 Copernicus Astronomical Center, Bartycka 18, 00-716 Warsaw, Poland \\ e-mail: yak@astro.ioffe.ru, ksen@astro.ioffe.ru, haensel@camk.edu.pl
}

Received 3 September 2002 / Accepted 17 April 2003

\begin{abstract}
We study thermal states of transiently accreting neutron stars (with mean accretion rates $\dot{M} \sim 10^{-14}-10^{-9} M_{\odot} \mathrm{yr}^{-1}$ ) determined by the deep crustal heating of accreted matter sinking into stellar interiors. We formalize a direct correspondence of this problem to the problem of cooling neutron stars. Using a simple toy model we analyze the most important factors which affect the thermal states of accreting stars: a strong superfluidity in the cores of low-mass stars and a fast neutrino emission (in nucleon, pion-condensed, kaon-condensed, or quark phases of dense matter) in the cores of high-mass stars. We briefly compare the results with the observations of soft X-ray transients in quiescence. If the upper limit on the quiescent thermal luminosity of the neutron star in SAX J1808.4-3658 (Campana et al. 2002) is associated with deep crustal heating, it favors the model of nucleon neutron-star cores with switched-on direct Urca process.
\end{abstract}

Key words. stars: neutron - dense matter

\section{Introduction}

Great progress in observations of soft X-ray transients (SXRTs) in quiescence has attracted attention to these objects. We consider the SXRTs containing neutron stars (NSs) in binary systems with low-mass companions (low-mass X-ray binaries); see Chen et al. (1997) for a review. They undergo periods of outburst activity (days-months, sometimes years) superimposed with periods of quiescence (months-decades). This transient activity is regulated most probably by the regime of accretion from the disks around the NSs. An active period is associated with switched-on accretion; the accretion energy released at the NS surface is high enough for a transient to look like a bright $\mathrm{X}$-ray source $\left(L_{\mathrm{X}} \sim 10^{36}-10^{38} \mathrm{erg} \mathrm{s}^{-1}\right)$. The accretion is switched off or strongly suppressed during quiescence intervals when the NS luminosity drops by several orders of magnitude $\left(L_{\mathrm{X}} \lesssim 10^{34} \mathrm{erg} \mathrm{s}^{-1}\right)$.

The nature of the quiescent emission is still a subject of debates. The hypothesis that this emission is produced by the thermal flux emergent from the NS interior has been rejected initially due to two reasons. First, the radiation spectra fitted by the blackbody model have given unreasonably small NS radii. Second, NSs in SXRTs have been expected to be internally cold; their quiescent emission should have been much lower than what is observed. These arguments were questioned by Brown et al. (1998). They suggested that the NSs can be warmed up to the required level by the deep crustal heating associated with nuclear transformations in accreted matter

Send offprint requests to: D. Yakovlev, e-mail: yak@astro.ioffe.ru sinking in the NS interiors (Haensel \& Zdunik 1990), while the radiation spectra can be fitted with the NS hydrogen atmosphere models (with realistic NS radii). It turned out that the emergent radiation flux may depend on the NS internal structure which opens an attractive possibility to explore the internal structure and the equation of state of dense matter by comparing the observations of SXRTs in quiescence with theoretical models (e.g., Ushomirsky \& Rutledge 2001; Colpi et al. 2001; Rutledge et al. 2002a; Brown et al. 2002, and references therein).

In this article, we discuss this possibility in more detail by making use of the close relationship between the theory of thermal states of transiently accreting NSs and the theory of cooling isolated NSs. Since the equation of state of NS matter is still poorly known we will make a general analysis of the problem with a simple toy model of NS thermal structure (described by Yakovlev \& Haensel 2003). This will enable us to confront the theory with the observations without performing complicated calculations.

\section{Numerical simulations}

Let us study the thermal state of a transiently accreting NS. Since the quiescence intervals are much shorter than typical time scales of NS thermal relaxation $\left(\sim 10^{4} \mathrm{yrs}\right.$, Colpi et al. 2001) we neglect short-term variability in the NS crust; it can be associated with variable residual accretion in quiescence, thermal relaxation of transient deep crustal heating, etc.; see, e.g., Ushomirsky \& Rutledge (2001) and Brown et al. (2002). Instead, we focus on a (quasi)stationary steady state of the NS 
determined by the mass accretion rate $\dot{M} \equiv\langle\dot{M}\rangle$ (from $10^{-14}$ to $10^{-9} M_{\odot} \mathrm{yr}^{-1}$ ) averaged over time intervals comparable with the thermal relaxation time scales. The accretion rates of study are too low to noticeably increase NS mass, $M$, during long periods of the NS evolution. The accreted material sinks gradually into the deep layers of the NS crust (into the density range from $\sim 10^{10} \mathrm{~g} \mathrm{~cm}^{-3}$ to $\sim 10^{13} \mathrm{~g} \mathrm{~cm}^{-3}$ ), where it undergoes various transformations (beta captures, neutron emissions and absorptions, pycnonuclear reactions) accompanied by a substantial energy release. This produces the deep crustal heating whose power is determined by $\dot{M}$. A complete sequence of nuclear transformations and associated energy release was calculated by Haensel \& Zdunik (1990) (see Bisnovatyi-Kogan 2001, for references to some earlier work). The total energy release is about $1.45 \mathrm{MeV}$ per accreting nucleon. The main energy release takes place at densities from about $10^{12}$ to $10^{13} \mathrm{~g} \mathrm{~cm}^{-3}$, about $1 \mathrm{~km}$ under the surface. The heating power is estimated as

$L_{\mathrm{dh}}=1.45 \mathrm{MeV} \dot{M} / m_{\mathrm{N}} \approx 8.74 \times 10^{33} \dot{M}_{10} \mathrm{erg} \mathrm{s}^{-1}$,

where $\dot{M}_{10} \equiv \dot{M} /\left(10^{-10} M_{\odot} \mathrm{yr}^{-1}\right)$ and $m_{\mathrm{N}}$ is the nucleon mass. Independently of the initial thermal state (cold or hot), a NS will eventually reach a stationary thermal state supported by this heating.

We stress that $L_{\mathrm{dh}}$ is much smaller than the mean energy release rate at the NS surface (where kinetic accretion energy transforms into heat) and in the outermost surface layers (at densities $\rho \lesssim 10^{10} \mathrm{~g} \mathrm{~cm}^{-3}$, where the accreted hydrogen or helium burns into heavier elements). We expect that the energy released at $\rho \lesssim 10^{10} \mathrm{~g} \mathrm{~cm}^{-3}$ is radiated away by the surface photon emission and does not heat the NS interior. This assumption is especially true for transiently accreting NSs, where the surface heat is rapidly emitted from the surface at the beginning of the quiescent stages.

The deep-heating theory of Haensel \& Zdunik (1990) assumes that the burning in the very surface layers leads to the production of iron-like elements at $\rho \sim 10^{10} \mathrm{~g} \mathrm{~cm}^{-3}$. According to the recent studies by Schatz et al. (2001) thermonuclear burning of hydrogen in the surface layers of accreting NSs may end at much heavier elements such as Sn, Sb and Te. The associated deep heating has been most recently analyzed by Haensel \& Zdunik (2003) with the result that the heavy elements in the surface layers do not significantly affect the composition of accreted matter and the energy release in the deeper layers of the inner crust (where the pycnonuclear reactions proceed); the total energy release remains about (1.1-1.5) MeV per nucleon.

To study the thermal states of accreting NSs we employ a simple toy model of cooling isolated NSs described by Yakovlev \& Haensel (2003). We have tested the results with those obtained with the "exact" codes which calculate the thermal states of cooling NSs and accreting NSs; the agreement has turned out to be satisfactory. Taking into account many theoretical and observational uncertainties, the toy model seems sufficient at the present stage of the investigation.

The toy model assumes that the NS core is divided into three zones: the outer zone, $\rho<\rho_{\mathrm{s}}$; the transition zone, $\rho_{\mathrm{s}} \leq$ $\rho<\rho_{\mathrm{f}}$; and the inner zone, $\rho \geq \rho_{\mathrm{f}}$. If the NS central density $\rho_{\mathrm{c}} \leq \rho_{\mathrm{s}}$, two last zones are absent.
Table 1. Main processes of slow neutrino emission in nucleon matter: Murca and bremsstrahlung (brems).

\begin{tabular}{|c|c|c|}
\hline Process & & 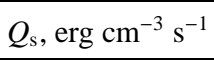 \\
\hline Murca & $\mathrm{nN} \rightarrow \mathrm{pNe} \bar{v} \quad \mathrm{pNe} \rightarrow \mathrm{nN} v$ & $10^{20}-3 \times 10^{21}$ \\
\hline Brems. & $\mathrm{NN} \rightarrow \mathrm{NN} v \bar{v}$ & $10^{19}-10^{20}$ \\
\hline
\end{tabular}

Table 2. Leading processes of fast neutrino emission in nucleon matter and three models of exotic matter.

\begin{tabular}{llll}
\hline \hline Model & Process & & $Q_{\mathrm{f}}, \mathrm{erg} \mathrm{cm}^{-3} \mathrm{~s}^{-1}$ \\
\hline Nucleon matter & $\mathrm{n} \rightarrow \mathrm{pe} \bar{v}$ & $\mathrm{pe} \rightarrow \mathrm{n} v$ & $10^{26}-10^{27}$ \\
Pion condensate & $\mathrm{q} \rightarrow \mathrm{qe} \bar{v}$ & $\mathrm{qe} \rightarrow \mathrm{q} v$ & $10^{23}-10^{26}$ \\
Kaon condensate & $\mathrm{q} \rightarrow \mathrm{qe} \bar{v}$ & $\mathrm{qe} \rightarrow \mathrm{q} v$ & $10^{23}-10^{24}$ \\
Quark matter & $\mathrm{d} \rightarrow \mathrm{ue} \bar{v}$ & $\mathrm{ue} \rightarrow \mathrm{d} \nu$ & $10^{23}-10^{24}$ \\
\hline
\end{tabular}

In the outer zone, the neutrino emission is supposed to be slow, while in the inner zone it is fast. The neutrino emissivity $Q_{v}\left(\mathrm{erg} \mathrm{s}^{-1} \mathrm{~cm}^{-3}\right)$ is assumed to be:

$Q_{v}^{\text {slow }}\left(\rho \leq \rho_{\mathrm{s}}\right)=Q_{\mathrm{s}} T_{9}^{8}, \quad Q_{v}^{\text {fast }}\left(\rho \geq \rho_{\mathrm{f}}\right)=Q_{\mathrm{f}} T_{9}^{6}$.

Here, $T_{9}$ is the internal stellar temperature $T$ expressed in $10^{9} \mathrm{~K}$, while $Q_{\mathrm{s}}$ and $Q_{\mathrm{f}}$ are constants. For simplicity, the toy model uses the linear interpolation in $\rho$ between $Q_{v}^{\text {slow }}$ and $Q_{v}^{\text {fast }}$ in the transition zone. The approximation is crude but sufficient for exploring the main unknown features of the transition zone: its position and thickness.

This generic description of $Q_{v}$ covers many physical models of nucleon and exotic supranuclear matter with different leading neutrino processes listed in Tables 1 and 2 (from Yakovlev \& Haensel 2003 with the kind permission of the authors). In these tables, $\mathrm{N}$ is a nucleon (neutron or proton, $\mathrm{n}$ or p); e is an electron; $v$ and $\bar{v}$ are neutrino and antineutrino; $\mathrm{q}$ is a quasinucleon (mixed $\mathrm{n}$ and $\mathrm{p}$ states); while $\mathrm{u}$ and $\mathrm{d}$ are quarks.

In particular, $Q_{\mathrm{s}}$ describes the modified Urca (Murca) process in nonsuperfluid nucleon matter, or weaker $\mathrm{NN}$ bremsstrahlung (e.g., nn-bremsstrahlung if Murca is suppressed by a strong proton superfluidity as considered by Kaminker et al. 2002). The factor $Q_{\mathrm{f}}$ describes the processes of fast neutrino emission: a very powerful direct Urca (Durca) process in nucleon matter (Lattimer et al. 1991) or somewhat weaker similar processes in exotic phases of matter (pion condensed, kaon condensed, or quark matter) as reviewed, e.g., by Pethick (1992). If hyperons appear in the NS core in addition to nucleons, the neutrino emissivity is expected to be qualitatively the same as in the nucleon core. The bottom line of Table 2 corresponds to nonsuperfluid quark matter in NS cores.

The transition zone models a switch-on of the fast neutrino emission with growing $\rho$. In nonsuperfluid matter, the lower density $\rho_{\mathrm{s}}$ is a threshold density of the fast emission; this threshold is usually sharp, i.e., $\rho_{\mathrm{s}} \approx \rho_{\mathrm{f}}$. The superfluidity suppresses the neutrino emission. Even if the superfluidity strength is high at the formal (nonsuperfluid) threshold, 
it decreases with growing $\rho$ in all realistic microphysical models of superfluid matter. In this case, $\rho_{\mathrm{s}}$ is the density where the superfluid suppression of the fast neutrino emission becomes weaker, while $\rho_{\mathrm{f}}$ is the density where this suppression almost vanishes. Thus the superfluidity can broaden a switch-on of the fast neutrino emission and produce a sufficiently wide transition zone. These effects are explained, e.g., by Yakovlev et al. (2002), taking nucleon Durca process and strong proton superfluidity as an example. In addition to reducing the ordinary neutrino emission processes, the superfluidity initiates a specific neutrino emission associated with Cooper pairing of baryons (Flowers et al. 1976). We neglect this emission in the present calculations but outline its consequences in Sect. 4 .

The toy model solves the equation of thermal balance of a cooling NS in the approximation of isothermal interior (see below) under a number of simplified assumptions on NS internal structure (Yakovlev \& Haensel 2003). The density profile in the star is approximate, $\rho(r)=\rho_{\mathrm{c}}\left(1-r^{2} / R^{2}\right)$, so that the NS mass is $M=8 \pi R^{3} \rho_{\mathrm{c}} / 15, R$ being the stellar radius. The stiffness of various equations of state can be mimicked by choosing different $M-R$ relations. For simplicity, following Yakovlev \& Haensel (2003), we set $R=12 \mathrm{~km}$ and vary $\rho_{\mathrm{c}}$ from $7 \times 10^{14}$ to $1.4 \times 10^{15} \mathrm{~g} \mathrm{~cm}^{-3}$, varying thus $M$ from $1.02 M_{\odot}$ to $2.04 M_{\odot}$. More realistic $M-R$ relations will not change our principal conclusions. Various cooling regimes are regulated by four parameters: $Q_{\mathrm{f}}, Q_{\mathrm{s}}, \rho_{\mathrm{s}}$, and $\rho_{\mathrm{f}}$, and are studied (Sect. 4) without complicated computation.

We have updated the toy model by incorporating the deep crustal heating as described below.

\section{Relation to cooling of isolated stars}

The thermal state of an accreting NS is similar to the state of a cooling isolated NS with isothermal interior.

Both states can be found by solving the equation of thermal balance of a NS with isothermal interior (e.g., Glen \& Sutherland 1980):

$C\left(T_{\mathrm{i}}\right) \frac{\mathrm{d} T_{\mathrm{i}}}{\mathrm{d} t}=L_{\mathrm{dh}}^{\infty}(\dot{M})-L_{v}^{\infty}\left(T_{\mathrm{i}}\right)-L_{\gamma}^{\infty}\left(T_{\mathrm{s}}\right)$,

where $T_{\mathrm{s}}$ is the effective surface temperature, $T_{\mathrm{i}}(t)=T(r, t) \mathrm{e}^{\Phi}$ is the redshifted internal temperature which is constant throughout the isothermal interior $\left(\rho \gtrsim \rho_{\mathrm{b}} \sim 10^{10} \mathrm{~g} \mathrm{~cm}^{-3}\right.$ ) with account for the effects of General Relativity; $T(r, t)$ is the local internal temperature of matter, and $\Phi(r)$ is the metric function. The relation between $T_{\mathrm{i}}$ and $T_{\mathrm{s}}$ is known from the solution of the thermal conduction problem in the outermost heat-blanketing stellar envelope $\left(\rho<\rho_{\mathrm{b}}\right)$. Furthermore, $C$ is the total heat capacity of the star, $L_{\gamma}^{\infty}=4 \pi \sigma T_{\mathrm{s}}^{4} R^{2}\left(1-r_{\mathrm{g}} / R\right)$ is the photon surface luminosity as detected by a distant observer $\left(r_{\mathrm{g}}=2 G M / c^{2}\right.$ being the gravitational radius), and

$L_{v, \mathrm{dh}}^{\infty}=4 \pi \int_{0}^{R} \frac{\mathrm{d} r r^{2} Q_{\nu, \mathrm{dh}} \mathrm{e}^{2 \Phi}}{\sqrt{1-2 G m /\left(c^{2} r\right)}}$,

are the redshifted neutrino luminosity and the redshifted power of the deep crustal heating; $Q_{v}$ is the neutrino emissivity; $Q_{\mathrm{dh}}$ is the energy release rate due to the deep crustal heating, and $m(r)$

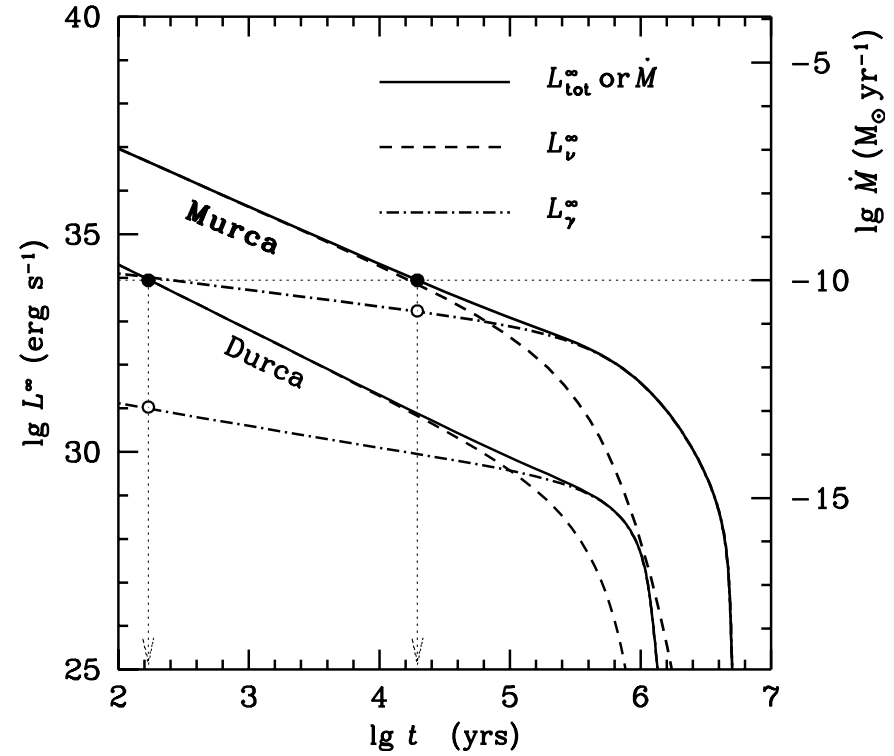

Fig. 1. Simultaneous calculation of cooling and heating curves for low-mass (thick lines) and high-mass (thin lines) NS models (see the text). Displayed are: the neutrino, photon and total luminosities, $L_{v}^{\infty}$, $L_{\gamma}^{\infty}$ and $L_{\text {tot }}^{\infty}$ (left vertical scale), of a cooling NS versus its age, and the accretion rate $\dot{M}$ (right vertical scale) of an accreting NS star with $L_{\mathrm{dh}}^{\infty}=L_{\mathrm{tot}}^{\infty}$. Dotted horizontal line: $L_{\mathrm{dh}}^{\infty}$ for $\dot{M}=10^{-10} M_{\odot} \mathrm{yr}^{-1}$. Filled circles and dotted vertical arrows refer to those low-mass and highmass cooling NSs which are equivalent to accreting NSs with the indicated $\dot{M}$; open circles: $L_{\gamma}^{\infty}$ for these cooling NSs.

is the gravitational mass contained in a sphere with radial coordinate $r$. In addition, we introduce the effective surface temperature detected by a distant observer, $T_{\mathrm{s}}^{\infty}=T_{\mathrm{s}} \sqrt{1-r_{\mathrm{g}} / R}$, and the "apparent" NS radius, $R^{\infty}=R / \sqrt{1-r_{\mathrm{g}} / R}$. Then $L_{\gamma}^{\infty}=4 \pi \sigma\left(T_{\mathrm{s}}^{\infty}\right)^{4}\left(R^{\infty}\right)^{2}$.

As mentioned above, we replace transient accretion by steady-state accretion with the mean constant accretion rate $\dot{M}=\langle\dot{M}\rangle$. We will study thus the steady states of NSs accreting at constant rates. Steady-state accretion in General Relativity is characterized by a constant mass accretion rate $\dot{M}$ which determines a constant number of accreting baryons passing through a sphere of any radial coordinate $r$ per unit time for a distant observer (e.g., Thorne \& Zytkow 1977). The gravitational redshift in a thin NS crust may be regarded as constant. In this approximation, sufficient for our problem, we have constant $\mathrm{e}^{\Phi}=$ $\sqrt{1-r_{\mathrm{g}} / R}$ throughout the crust, and $L_{\mathrm{dh}}^{\infty}=L_{\mathrm{dh}} \sqrt{1-r_{\mathrm{g}} / R}$, where $L_{\mathrm{dh}}$ is given by Eq. (1) (neglecting the effects of General Relativity). In principle, the gravitational redshift has to be included into $L_{\mathrm{dh}}^{\infty}$. However, since $\dot{M}$ in SXRTs is determined with large uncertainties, it is premature to include such effects, and we set $L_{\mathrm{dh}}^{\infty}=L_{\mathrm{dh}}$ in subsequent calculations.

Following the cooling of an isolated NS we calculate the cooling curve, the dependence of the surface temperature or the photon surface luminosity on NS age, $T_{\mathrm{s}}^{\infty}(t)$ or $L_{\gamma}^{\infty}(t)$. In about $t \sim 100 \mathrm{yrs}$ (when $T_{\mathrm{s}}^{\infty}$ drops below $3 \times 10^{6} \mathrm{~K}$ ) the NS interior becomes isothermal. Subsequent cooling can be considered as a sequence of quasistationary states. It is described by Eq. (3) with $L_{\mathrm{dh}}^{\infty}=0$. The examples are presented in Fig. 1 described below. 


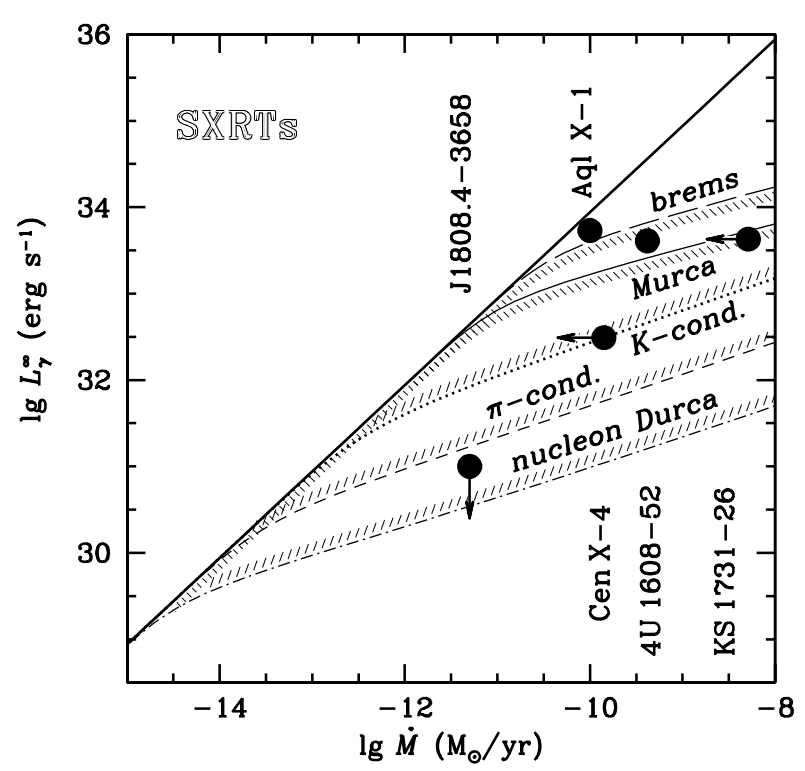

Fig. 2. Heating curves of accreting NSs compared with observations of several SXRTs in quiescence. Thick solid line: deep crustal heating power, $L_{\mathrm{dh}}$. Long dashes and thin solid lines: two models of heating curves of low-mass NSs $\left(Q_{\mathrm{s}}=3 \times 10^{19}\right.$ and $\left.10^{21}\right)$. Dotted, shortdashed, and dot-and-dashed lines: three models of high-mass NSs $\left(Q_{\mathrm{f}}=10^{23}, 10^{25}\right.$, and $\left.10^{27}\right)$.

Following the evolution of an accreting NS from Eq. (3), we would find that the NS, starting from an arbitrary initial thermal state, eventually reaches a stationary state supported by the deep crustal heating (whose power, $L_{\mathrm{dh}}^{\infty}$, is given by Eq. (1)). The steady state is reached when the total NS luminosity, $L_{\text {tot }}^{\infty}=$ $L_{v}^{\infty}+L_{\gamma}^{\infty}$, is balanced by $L_{\mathrm{dh}}^{\infty}$. This steady state is described by Eq. (3) in the stationary limit:

$L_{\mathrm{dh}}^{\infty}(\dot{M})=L_{v}^{\infty}\left(T_{\mathrm{i}}\right)+L_{\gamma}^{\infty}\left(T_{\mathrm{s}}\right)$,

where $L_{\mathrm{dh}}^{\infty}$ is known once $\dot{M}$ is specified. Since we are interested in the steady state, it is sufficient to solve Eq. (5). The solutions give us a heating curve, the dependence of the surface temperature or the photon luminosity on the mean accretion rate, $T_{\mathrm{s}}^{\infty}(\dot{M})$ or $L_{\gamma}^{\infty}(\dot{M})$. For the values of $\dot{M} \lesssim 10^{-9} M_{\odot} \mathrm{yr}^{-1}$, we obtain the thermal states with $T_{\mathrm{s}} \lesssim 3 \times 10^{6} \mathrm{~K}$. The examples are presented in Fig. 2 discussed below.

Comparing the equations of NS cooling and accretion heating we see that any steady state of a NS accreting at a rate $\dot{M}$ is equivalent to a state of a cooling NS in such a moment of time $t$ when $L_{\text {tot }}^{\infty}(t)=L_{\mathrm{dh}}^{\infty}(\dot{M})$. In other words, calculating a cooling curve, we find $L_{\text {tot }}^{\infty}(t), L_{\gamma}^{\infty}(t)$ and equivalent $\dot{M}(t)$ for any moment of time. Considering $t$ as a parameter, we immediately get a heating curve $L_{\gamma}^{\infty}(\dot{M})$.

This procedure is illustrated in Fig. 1. It shows a simultaneous solution of the cooling and heating problems with the toy model (Sect. 2) which calculates the cooling of isolated low-mass and high-mass NSs. The masses are 1.16 and $2.04 M_{\odot}\left(\rho_{\mathrm{c}}=8 \times 10^{14}\right.$ and $\left.1.4 \times 10^{15} \mathrm{~g} \mathrm{~cm}^{-3}\right)$. The toymodel parameters are: $Q_{\mathrm{s}}=10^{21} \mathrm{erg} \mathrm{cm}^{-3} \mathrm{~s}^{-1}$ and $Q_{\mathrm{f}}=$ $10^{27} \mathrm{erg} \mathrm{cm}^{-3} \mathrm{~s}^{-1}$ (appropriate to the Murca and Durca processes in nonsuperfluid matter), $\rho_{\mathrm{s}}=8 \times 10^{14} \mathrm{~g} \mathrm{~cm}^{-3}$, and $\rho_{\mathrm{f}}=$ $10^{15} \mathrm{~g} \mathrm{~cm}^{-3}$. The figure shows the decrease of $L_{\gamma}^{\infty}, L_{v}^{\infty}$, and $L_{\text {tot }}^{\infty}$ (left vertical axis) with time for cooling stars. The right vertical axis presents $\dot{M}$ for accreting NSs with $L_{\mathrm{tot}}=L_{\mathrm{dh}}$. For instance, taking $\dot{M}=10^{-10} M_{\odot} \mathrm{yr}^{-1}$ (horizontal dotted line) and locating the points, where $L_{\mathrm{tot}}=L_{\mathrm{dh}}$ (filled circles and vertical dotted arrows), we find that the low-mass NS accreting with the given $\dot{M}$ is equivalent to the cooling NS of age $t \approx 2 \times 10^{4} \mathrm{yrs}$, while the high-mass accreting NS is equivalent to the cooling NS of age $t \approx 170 \mathrm{yrs}$. The thermal states of low-mass and high-mass accreting NSs are seen to be drastically different (the appropriate photon luminosities $L_{\gamma}^{\infty}$ are marked by open circles).

It is clear that any steady state of an accreting NS is independent of the heat capacity of matter and the thermal conductivity in the isothermal NS interior, although the relaxation to this state does depend on these quantities. Accordingly, superfluidity in NS interiors affects the steady state only by regulating the neutrino emission processes.

Although a general analysis of Eq. (5) is complicated, we can mention two limiting regimes.

(i) The photon emission regime is realized in cold enough NSs, where $L_{v}^{\infty} \ll L_{\gamma}^{\infty}$, i.e., the heat released in the deep crust is carried away by the thermal conductivity to the surface and emitted in the form of surface photon radiation. This regime is equivalent to the photon emission stage of cooling NSs. In this regime, Eq. (5) reduces to: $L_{\mathrm{dh}}^{\infty}(\dot{M})=L_{\gamma}^{\infty}\left(T_{\mathrm{s}}\right)$, which immediately gives an estimate $T_{\mathrm{s} 6} \equiv T_{\mathrm{s}} / 10^{6} \mathrm{~K} \sim 2 \dot{M}_{10}^{1 / 4}$ (for $R \sim 10 \mathrm{~km}$ ). Accordingly, the surface temperature is determined by the accretion rate, and it is independent of the internal structure of the NS. The internal temperature can be found then using the $T_{\mathrm{s}}-T_{\mathrm{i}}$ relation. For typical surface gravities $g_{\mathrm{s}} \sim$ $2 \times 10^{14} \mathrm{~cm} \mathrm{~s}^{-2}$ from the relation of Gudmundsson et al. (1983) we have $T_{\mathrm{i} 9} \equiv T_{\mathrm{i}} / 10^{9} \mathrm{~K} \sim 0.1 T_{\mathrm{s} 6}^{2}$. Thus, $T_{\mathrm{i} 9} \sim 0.4 \sqrt{\dot{M}_{10}}$.

(ii) The neutrino emission regime is realized in warmer NSs, where $L_{v}^{\infty} \gg L_{\gamma}^{\infty}$, i.e., the heat released in the deep crust is spread by thermal diffusion over the star and carried away by neutrino emission. This regime is equivalent to the neutrino stage in cooling NSs. The thermal balance equation then reads: $L_{\mathrm{dh}}^{\infty}(\dot{M})=L_{v}^{\infty}\left(T_{\mathrm{i}}\right)$, which gives $T_{\mathrm{i}}$. The surface temperature can be found then using the $T_{\mathrm{s}}-T_{\mathrm{i}}$ relation. In this regime, the surface temperature $T_{\mathrm{s}}$ does depend on the internal structure of the star, on the equation of state, the presence or absence of an enhanced neutrino emission mechanism, and on the nature of this emission. For the assumptions implied in our toy model, we have two distinctly different cases.

(ii.a) In low-mass NSs $\left(\rho_{\mathrm{c}} \leq \rho_{\mathrm{s}}\right)$ we have slow neutrino emission, $Q_{v}^{\text {slow }}=Q_{\mathrm{s}} T_{\mathrm{i} 9}^{8}$. Introducing $Q_{\mathrm{s} 21} \equiv$ $Q_{\mathrm{s}} /\left(10^{21} \mathrm{erg} \mathrm{s}^{-1} \mathrm{~cm}^{-3}\right)$, we have $L_{v} \sim 5 \times 10^{39} T_{\mathrm{i} 9}^{8} Q_{\mathrm{s} 21} \mathrm{erg} \mathrm{s}^{-1}$. Then we obtain $T_{\mathrm{i} 9} \sim 0.18\left(\dot{M}_{10} / Q_{\mathrm{s} 21}\right)^{1 / 8}$, and $T_{\mathrm{s} 6} \sim$ $1.4\left(\dot{M}_{10} / Q_{\mathrm{s} 21}\right)^{1 / 16}$; the surface temperature weakly depends on the accretion rate and the neutrino emission level $Q_{\text {s }}$ (as a result of the strong temperature dependence of $L_{v}$ ). The comparison with case (i) shows that the transition from the photon emission regime to the neutrino emission regime takes place at $\dot{M}_{10} \sim 0.13 / Q_{\mathrm{s} 21}^{1 / 3}$ and $T_{\mathrm{s} 6} \sim 1.2 / Q_{\mathrm{s} 21}^{1 / 12}$.

(ii.b) In high-mass $N S s\left(\rho_{\mathrm{c}}>\rho_{\mathrm{f}}\right)$ the neutrino emission is mainly produced by the fast mechanism, $Q_{v}^{\text {fast }}=Q_{\mathrm{f}} T_{\mathrm{i} 9}^{6}$, in the inner zone $\left(\rho>\rho_{\mathrm{f}}\right)$ of the NS core. Introducing $Q_{\mathrm{f} 27} \equiv$ $Q_{\mathrm{f}} /\left(10^{27} \mathrm{erg} \mathrm{s}^{-1} \mathrm{~cm}^{-3}\right)$, we have $L_{v} \sim 5 \times 10^{45} T_{\mathrm{i} 9}^{6} Q_{\mathrm{f} 27} \mathrm{erg} \mathrm{s}^{-1}$, 
Table 3. Parameters of NSs in SXRTs.

\begin{tabular}{lcccccl}
\hline \hline Source & $\dot{M}, M_{\odot} \mathrm{yr}^{-1}$ & $L_{\gamma}^{\infty}, \mathrm{erg} \mathrm{s}^{-1}$ & $T_{\mathrm{s}}^{\infty}, \mathrm{eV}$ & $R^{\infty}, \mathrm{km}$ & \multicolumn{2}{c}{ Reference } \\
\hline Aq1 X-1 & $1.0 \times 10^{-10}$ & $5.3 \times 10^{33}$ & 113 & 15.9 & Rutledge et al. (2002b): & Table 6, fit 10 \\
Cen X-4 & $1.4 \times 10^{-10}$ & $3.1 \times 10^{32}$ & $76^{*}$ & 12.9 & Rutledge et al. (2001): & Table 4 \\
4U 1608-522 & $4.2 \times 10^{-10}$ & $4.1 \times 10^{33}$ & $170^{*}$ & 9.4 & Rutledge et al. (1999): & Table 22 \\
KS 1731-260 & $5.1 \times 10^{-9}$ & $4.3 \times 10^{33}$ & 110 & 15 & Wijnands et al. (2002): & Table 1, fit 2 \\
SAX J1808.4-3658 & $5.0 \times 10^{-12}$ & $\$ 1.0 \times 10^{31}$ & - & - & Campana et al. (2002): & Sect. 2.2 \\
\hline
\end{tabular}

* Nonredshifted.

$T_{\mathrm{i} 9} \sim 0.01\left(\dot{M}_{10} / Q_{\mathrm{f} 27}\right)^{1 / 6}$, and $T_{\mathrm{s} 6} \sim 0.3\left(\dot{M}_{10} / Q_{\mathrm{f} 27}\right)^{1 / 12} ; T_{\mathrm{s}}$ is again a weak function of $\dot{M}$ and $Q_{\mathrm{f}}$. A comparison with case (i) shows that the transition between the photon and neutrino emission regimes occurs at $\dot{M}_{10} \sim 10^{-5} / \sqrt{Q_{\mathrm{f} 27}}$ and $T_{\mathrm{s} 6} \sim 0.1 / Q_{\mathrm{f} 27}^{1 / 8}$. We have a much colder NS than in case (ii.a), and the neutrino emission regime persists to much lower $\dot{M}$.

\section{Results and discussion}

Our numerical calculations with the toy model confirm this qualitative analysis. The results are presented in Fig. 2 which shows the surface photon luminosity versus accretion rate for low-mass and high-mass NSs.

The thick solid curve presents the deep heating power, $L_{\mathrm{dh}}^{\infty}$, which is the upper limit of $L_{\gamma}^{\infty}$ for any accreting source.

Moving from top to bottom, the next two lines refer to lowmass NSs with two types of slow neutrino emission appropriate either to neutron-neutron bremsstrahlung in the NS cores with a strong proton superfluidity $\left(Q_{\mathrm{s}}=3 \times 10^{19}\right)$ or to the Murca process in nonsuperfluid cores $\left(Q_{\mathrm{s}}=10^{21}\right)$. The strong proton superfluidity damps the Murca process and enables us to obtain hotter NSs, just as in the theory of cooling NSs (e.g., Kaminker et al. 2002).

The three next lines refer to high-mass NSs with three types of fast neutrino emission appropriate to (from top to bottom) kaon-condensed matter $\left(Q_{\mathrm{f}}=10^{23}\right)$, pion-condensed matter $\left(Q_{\mathrm{f}}=10^{25}\right)$, or nucleon matter with open Durca process $\left(Q_{\mathrm{f}}=10^{27}\right)$. NSs with hyperon cores are expected to cool at about the same rate as NSs with nucleon cores.

The heating curves of low-mass NSs provide the upper limit of $L_{\gamma}^{\infty}$, while the curves of high-mass stars give the lower limit of $L_{\gamma}^{\infty}$, for any particular equation of state (EOS) of NS interiors (for any set of the four neutrino emission parameters, in our case). Accordingly, the upper limits are shaded below the curves, and the lower limits are shaded above the curves. As in the cooling theory (Yakovlev \& Haensel 2003), the upper and lower heating curves are almost insensitive to the position and width of the transition zone if $\rho_{\mathrm{f}}$ and $\rho_{\mathrm{s}}$ are located anywhere between $8 \times 10^{14}$ and $1.2 \times 10^{15} \mathrm{~g} \mathrm{~cm}^{-3}$. The upper curve is determined by the parameter $Q_{\mathrm{s}}$, while the lower one is determined by $Q_{\mathrm{f}}$.

Varying the NS mass from the lowest values to the highest we obtain a family of heating curves which fill in the space in Fig. 2 between the upper and lower curve for a given EOS of dense matter. The group of NSs whose heating curves lie essentially between the upper and lower curves will be called medium-mass stars. Their central density falls within the transition layer between the slow and fast neutrino emission zones ( $\rho_{\mathrm{s}} \lesssim \rho \lesssim \rho_{\mathrm{f}}$, Sect. 2), and their mass range is sensitive to the position and width of this layer. The same situation occurs in the theory of cooling NSs (e.g., Yakovlev \& Haensel 2003). Thus, for a given EOS of dense matter we obtain its own upper and lower heating curves, and intermediate heating curves of medium-mass stars.

In analogy with the cooling theory, the existence of a representative class of medium-mass stars (a smooth transition from the upper to the lower heating curves with increasing $M$ ) depends on the relative width of the transition zone, $\Delta \rho / \rho_{\mathrm{s}} \equiv$ $\left(\rho_{\mathrm{f}}-\rho_{\mathrm{s}}\right) / \rho_{\mathrm{s}}$, and on the contrast between the fast and slow neutrino emissivities, $Q_{\mathrm{f}} / Q_{\mathrm{s}}$. To ensure the existence of this NS class for a sharp emissivity contrast, $Q_{\mathrm{f}} / Q_{\mathrm{s}} \gg 10^{3}$, we need a rather wide transition zone, $\Delta \rho / \rho_{\mathrm{s}} \gtrsim 0.1$. On the other hand, this class will be available even for a negligibly narrow zone if the emissivity contrast is lower, $Q_{\mathrm{f}} / Q_{\mathrm{s}} \lesssim 10^{3}$.

These results can be confronted with the observations of SXRTs containing NSs. Although our theoretical toy model is oversimplified, in Fig. 2 we present an example of such an analysis for five SXRTs: Aql X-1, Cen X-4, 4U 1608-522, KS 1731-26, and SAX J1808.4-3658. The data are rather uncertain. Thus we plot the observational points as thick dots.

The parameters of the selected sources are collected in Table 3. The mean mass accretion rate $\dot{M}$ is evaluated as $\dot{M}=\Delta M / \Delta t$, where $\Delta M$ is the total mass accreted over a representative period of time $\Delta t$. Both $\Delta M$ and $\Delta t$ should include active and quiescent periods, although $\Delta M$ is mainly accumulated in the outburst states. In principle, we need $\dot{M}$ averaged over thermal relaxation time scales, $\sim 10^{4}$ yrs, while the observations provide us with sparse data over periods not longer than several decades. For Aql X-1, the mean $\dot{M}$ has been estimated by Rutledge et al. (2000) (their Sect. 5, an estimate from the RXTE/ASM light curve history). For Cen X-4 and 4U 1608522, we obtain $\dot{M}$ from Table 9 in Chen et al. (1997). Following these authors, we take $\Delta t=8.67$ yrs for $4 \mathrm{U} 1608-522$, which is a frequently bursting source $(\Delta M$ is estimated for 6 outbursts in the period from 1970 to 1979). We take $\Delta t=33.16 \mathrm{yrs}$ for Cen X-4 (with $\Delta M$ given for the only two outbursts in 1969 and 1979), adding the period from 1979 till now when no outbursts were observed. Since the active states may be very rare for this source, it is safer to consider the obtained $\dot{M}$ as an 
upper limit. KS 1731-260 recently (about 1.5 years ago) returned into quiescence after having actively accreted for $\approx 11.5$ years. For this source, we take $\Delta t=11.5+1.5=$ 13 yrs. We estimate the mass $\Delta M$ accreted during the long outburst state from the value of the mean outburst flux given by Rutledge et al. (2002a, Sect. 3.1). The estimation is made in the same manner as in Chen et al. (1997, Sect. 5.1.4). Since the recurrence time is unknown, our value of $\dot{M}$ is most probably an upper limit. Finally, $\dot{M}$ for SAX J1808.4-3658 was estimated by Bildstein \& Chakrabarty (2001) and Campana et al. (2002).

The values of $L_{\gamma}^{\infty}$ in Fig. 2 are meant to refer to the quiescent thermal luminosity from the NS surfaces. For the first four sources, these values are obtained from the values of $T_{\mathrm{s}}^{\infty}$ and $R^{\infty}$ given in Table 3. The values of $T_{\mathrm{s}}^{\infty}$ and $R^{\infty}$ were evaluated by the authors cited in Table 3 by fitting the observed spectra with the hydrogen atmosphere models. Note that the values of the surface temperature for Cen X-4 and 4U 1608-522 are nonredshifted. We have redshifted them assuming $M=1.4 M_{\odot}$ and $R=12 \mathrm{~km}$. The spectrum of SAX J1808.4-3658 in quiescence is well fitted by a power law, i.e., no surface thermal emission has been detected. The estimates of the upper limit of $L_{\gamma}^{\infty}$ are model dependent and range from about $10^{30} \mathrm{erg} \mathrm{s}^{-1}$ to $2.5 \times 10^{31} \mathrm{erg} \mathrm{s}^{-1}$ (Campana et al. 2002). We take $L_{\gamma}^{\infty}=$ $10^{31} \mathrm{erg} \mathrm{s}^{-1}$ with the notice that the actual surface luminosity may be much lower.

If the interpretation of quiescent emission as the thermal emission from the NS surfaces is correct, then all five NSs are heated to the neutrino emission stage $\left(L_{\mathrm{dh}}^{\infty}>L_{\gamma}^{\infty}\right)$. Since $L_{\mathrm{dh}}^{\infty}$ is reliably determined by the theory (Haensel \& Zdunik 1990, 2003) for a known $\dot{M}$, and $L_{\gamma}^{\infty}$ is measured, one can immediately estimate the neutrino luminosity of any source from the thermal balance, Eq. (5): $L_{v}^{\infty}=L_{\mathrm{dh}}^{\infty}-L_{\gamma}^{\infty}$. In all our cases $L_{v}^{\infty}$ is comparable with $L_{\mathrm{dh}}^{\infty}$ (Fig. 2).

As seen from Fig. 2, we can treat NSs in 4U 1608-52 and Aql X-1 as low-mass NSs with very weak neutrino emission from their cores (suppressed by strong nucleon superfluidity). The NSs in Cen X-4 and SAX J1808.4-3658 seem to require enhanced neutrino emission and are thus more massive. The status of the NS in KS 1731-26 is less certain because of poorly determined $\dot{M}$ (see above). If the real value of $\dot{M}$ is close to that in Table 3 it may also require some enhanced neutrino emission. Similar conclusions have been made by several authors (particularly, by Ushomirsky \& Rutledge 2001; Colpi et al. 2001; Rutledge et al. 2001, 2002a, 2002b; Brown et al. 2002; Wijnands et al. 2002) with respect to some of these sources or selected groups. Colpi et al. (2001) presented also the heating curves for specific models of low-mass and highmass NSs with superfluid nucleon cores and suggested that by tuning nucleon superfluidity and NS masses one can explain the data. Using the toy-model, we can present a general analysis of the problem for different EOSs of NS interiors (assuming, of course, that all the sources have to be interpreted in terms of one EOS). In this way we can quantify the assumptions on enhanced neutrino emission in terms of pion condensed, kaon condensed, and Durca-allowed nucleon models of matter.

Disregarding the SAX source for the moment, we can treat the NS in Cen X-4 either as a high-mass NS (with a kaon-condensed or quark core) or as a medium-mass NS (with a pion-condensed, quark, or Durca-allowed nucleon core); thus we cannot determine the nature of superdense matter. If the data on SAX J1808.4-3658 are really relevant for our analysis we only have the choice to treat the NS as a high-mass NS with the nucleon core (and the NS in Cen X-4 as the medium-mass NS with the nucleon core). This would mean that NS cores do not contain exotic phases of matter.

Our toy model is too flexible and does not allow us to fix the position of the transition layer in the stellar cores where the slow neutrino emission transforms into the fast one. Adopting a specific EOS of NS interiors (with this position determined by microphysics input) we would be able to construct the sequences of heating curves for the stars with different $M$, and attribute certain values of $M$ to any source ("weigh" NSs in SXRTs, as proposed by Colpi et al. 2001, just as in the case of cooling isolated NSs considered, e.g., by Kaminker et al. 2002). We intend to do this in our future publications, using an exact cooling code and taking into account some effects neglected in our simplified approach.

In particular, we will account for the presence of light elements on the NS surfaces: they change the thermal conductivity of the NS heat-blanketing envelope and the relation between the surface and internal temperature of NSs. The effect is well known for cooling NSs (Potekhin et al. 1997) and has been applied recently to accreting NSs (Brown et al. 2002).

We will also carefully treat the effects of baryon superfluidity in NS interiors (just as for cooling NSs, see, e.g., Kaminker et al. 2002). Particularly, we will study the effects of Cooper-pairing neutrino emission of baryons neglected in the present analysis. Under certain conditions, this neutrino emission would violate our general assumptions on the neutrino emissivity $Q_{v}(T, \rho)$ and complicate our study. For instance, according to our estimates, the ${ }^{3} \mathrm{P}_{2}$ neutron superfluidity with the maximum values of the density dependent critical temperature $T_{\mathrm{cn}}(\rho)$ from $\sim 10^{8} \mathrm{~K}$ to $\sim 2 \times 10^{9} \mathrm{~K}$ in the nucleon NS core would produce powerful Cooper pairing neutrino emission and strongly affect the thermal states of accreting NSs. However, the same effect would initiate a really fast cooling of not too massive isolated NSs in contradiction with the observations of isolated cooling NSs (e.g., Kaminker et al. 2002). Thus the presence of the indicated neutron superfluidity can be rejected on these grounds.

Because of the similarity between the heating and cooling curves, the observations of cooling isolated NSs and accreting NSs in SXRTs can be analyzed together employing the same EOSs of NS interiors. This increases the statistics of the sources and the confidence of the results. The theory of cooling NSs has recently been confronted with observations by Yakovlev \& Haensel (2003). Some cooling NSs (first of all, RX J0822-43 and PSR 1055-52) can be interpreted (Kaminker et al. 2002) as low-mass NSs with strong proton superfluidity in their cores. Other sources (first of all, Vela and Geminga) seem to require fast neutrino emission but the nature of this emission (a choice of fast-cooling model from Table 2) is uncertain, just as for SXRTs disregarding the data on SAX J1808.4-3658. In this context, the latter source is now the only one which indicates the absence of exotic phases of matter in NS cores. 
The assumption that the observed X-ray emission of SXRTs in quiescence emerges from the NS interior is still an attractive hypothesis. In any case the theory of deep crustal heating is solid and leaves no doubts that this heating does occur in accreting NSs leading to observational consequences.

Acknowledgements. The authors are grateful to anonymous referee for critical comments and to J. L. Zdunik for new calculations of the energy release in the deep crustal heating. KPL and DGY acknowledge hospitality of N. Copernicus Astronomical Center in Warsaw. This work was supported in part by the RBRF (grants Nos. 02-0217668 and 03-07-90200) and KBN (grant 5 P03D 020 20).

\section{References}

Bildsten, L., \& Chakrabarty, D. 2001, ApJ, 557, 292

Bisnovatyi-Kogan, G. S. 2001, Stellar Physics (Berlin: Springer), vols. 1 and 2

Brown, E. F., Bildsten, L., \& Rutledge, R. E. 1998, ApJ, 504, L95

Brown, E. F., Bildsten, L., \& Chang, P. 2002, ApJ, 574, 920

Campana, S., Stella, L., Gastaldello, F., et al. 2002, ApJ, 575, L15

Colpi, M., Geppert, U., Page, D., \& Possenti, A. 2001, ApJ, 548, L175

Chen, W., Shrader, C. R., \& Livio, M. 1997, ApJ, 491, 312

Flowers, E. G., Ruderman, M., \& Sutherland, P. G. 1976, ApJ, 205,541

Glen, G., \& Sutherland, P. 1980, ApJ, 239, 671

Gudmundsson, E. H., Pethick, C. J., \& Epstein, R. I. 1983, ApJ, 272,286
Haensel, P., \& Zdunik, J. L. 1990, A\&A, 227, 431

Haensel, P., \& Zdunik, J. L. 2003, A\&A, 404, L33

Kaminker, A. D., Yakovlev, D. G., \& Gnedin, O. Y. 2002, A\&A, 383, 1076

Lattimer, J. M., Pethick, C. J., Prakash, M., \& Haensel, P. 1991, Phys. Rev. Lett., 66, 2701

Pethick, C. J. 1992, Rev. Mod. Phys., 64, 1133

Potekhin, A. Y., Chabrier, G., \& Yakovlev, D. G. 1997, A\&A, 323, 415

Rutledge, R. E., Bildsten, L., Brown, E. F., Pavlov, G. G., \& Zavlin, V. E. 1999, ApJ, 514, 945

Rutledge, R. E., Bildsten, L., Brown, E. F., Pavlov, G. G., \& Zavlin, V. E. 2000, ApJ, 529, 985

Rutledge, R. E., Bildsten, L., Brown, E. F., Pavlov, G. G., \& Zavlin, V. E. 2001, ApJ, 551, 921

Rutledge, R. E., Bildsten, L., Brown, E. F., et al. 2002a, ApJ, 580, 413

Rutledge, R. E., Bildsten, L., Brown, E. F., Pavlov, G. G., \& Zavlin, V. E. 2002b, ApJ, 477, 346

Schatz, H., Aprahamian, A., Barnard, V., et al. 2001, Phys. Rev. Lett. 86,3471

Thorne, K. S., \& Zytkow, A. N. 1977, ApJ, 212, 832

Ushomirsky, G., \& Rutledge, R. E. 2001, MNRAS, 325, 1157

Wijnands, R., Guainazzi, M., van der Klis, M., \& Méndez, M. 2002, ApJ, 573, L45

Yakovlev, D. G., Gnedin, O. Y., Kaminker, A. D., \& Potekhin, A. Y. 2002, in Proc. of 270 Heraeus Seminar on Neutron Stars, Pulsars and Supernova Remnants, ed. W. Becker, H. Lesh, \& J. Trümper (Garching: MPE), 287

Yakovlev, D. G., \& Haensel, P. 2003, A\&A, 407, 259 